\title{
Effets de la durée de conservation et du séchage sur la teneur en azote des fourrages pauvres traités à l'urée
}

\author{
H. Nantoumé ${ }^{1}$ A. Kouriba ${ }^{1}$ B. Ouologuem ${ }^{2}$
}

Mots-clés

Fourrage - Teneur en azote - Urée Aptitude à la conservation - Séchage Qualité - Mali.

\begin{abstract}
Résumé
Dans le cadre d'une expérimentation de 90 jours conçue pour étudier les effets de la durée de conservation et du séchage sur la teneur en azote de fourrages pauvres (paille de brousse, chaumes de sorgho, chaumes de maïs et chaumes de mil) traités à 4,5 p. 100 d'urée, des bocaux ont été utilisés comme silos de laboratoire. La teneur en azote des fourrages traités est restée significativement plus élevée $(P<0,05)$ que celle des fourrages non traités, quelle qu'ait été la durée de conservation. Le séchage a diminué la teneur en azote $(\mathrm{P}<0,05)$ des fourrages traités. Une fois le traitement effectif (15 jours), la teneur en azote des fourrages traités n'a pas varié $(p>0,05)$ au cours de I'essai. L'étude a montré qu'il était plus avantageux d'offrir les fourrages traités non séchés pour minimiser les pertes d'azote au cours du séchage.
\end{abstract}

\section{INTRODUCTION}

Pendant la longue saison sèche, la majeure partie des aliments du bétail de la région de Kayes est constituée par la paille de brousse et les résidus de culture. L'utilisation digestive de ces aliments est réduite à cause de leur teneur élevée en composés ligno-cellulosiques et de leur faible teneur en azote. Plusieurs traitements (physiques, chimiques et biologiques) ont été utilisés pour améliorer la valeur alimentaire des fourrages pauvres.

Parmi les agents chimiques utilisés pour le traitement des fourrages pauvres, l'ammoniac a reçu une attention considérable. L'ammoniac améliore la valeur alimentaire des fourrages pauvres vraisemblablement en solubilisant l'hémicellulose et en gonflant la cellulose avec comme effet l'amélioration de la digestibilité et de l'ingestibilité de la matière sèche (4). De plus, l'azote qui est ajouté au fourrage comme résultat de traitement est utilisé par la population microbienne du rumen quand suffisamment d'énergie est disponible (7). L'urée a été proposée comme source d'ammoniac pour l'amélioration de la qualité des fourrages pauvres (1).

1. Centre régional de recherche agronomique de Kayes, BP 281, Kayes, Mali 2. Centre régional de recherche agronomique de Sotuba, BP 262, Bamako, Mali
Des études entreprises au Mali sur l'amélioration de la valeur alimentaire des fourrages pauvres ont indiqué une augmentation de l'ingestibilité et de la digestibilité du fourrage traité $(2,3,6)$. La plupart de ces études ont été faites sur la paille de riz. Les données sur les traitements à l'urée des autres fourrages pauvres sont rares. L'influence de la durée de conservation et du séchage sur la teneur en azote, peu abordée dans la littérature, a été l'objet de cette étude.

\section{MATERIEL ET METHODES}

Deux essais ont été conduits. Le premier a porté sur la paille de brousse et les chaumes de sorgho. La paille de brousse, en provenance des alentours de la ville de Kayes, a été essentiellement composée de Schoenefeldia gracilis et de Schizachirium exile. Les chaumes de sorgho (Sorghum bicolor) sont provenus des exploitations agricoles locales. Les travaux (traitement à l'urée et analyses chimiques) ont été effectués au Laboratoire de nutrition animale de Sotuba à Bamako où les moyennes des températures minimales et maximales de 40 ans ont été respectivement de 21,7 et $34,7^{\circ} \mathrm{C}(5)$. Chaque échantillon de fourrage $(4,5 \mathrm{~kg})$ a été traité avec $0,2 \mathrm{~kg}$ d'urée dissoute dans $5 \mathrm{~kg}$ d'eau, soit à 4,5 p. 100. La méthode utilisée a été celle décrite par Hadjipanayiotou (1). Au cours du premier essai, 42 bocaux (d'une capacité de 0,51 chacun) ont été remplis de fourrages traités (21 bocaux par fourrage), fermés 
étanchement et conservés au Laboratoire de nutrition animale de Sotuba. Ces fourrages ont fait l'objet d'analyses chimiques après $1,15,30,45,60,75$ et 90 jours de conservation. Des échantillons prélevés dans trois bocaux de chaque fourrage pris au hasard ont été analysés après un jour de conservation, puis tous les 15 jours.

Les analyses chimiques ont porté sur la teneur en matière sèche (MS) et en azote total. L'azote a été déterminé sur les échantillons frais et séchés pendant trois jours à l'abri du soleil.

L'analyse de variance a été utilisée avec un dispositif factoriel de 2 (types de fourrages) * 2 (états ; humide et séché) $* 7$ (durée de conservation). Pour des effets significatifs observés, la séparation des moyennes à été faite par la méthode de la plus petite différence significative (Ppds). Le modèle utilisé a été le suivant :

$\mathrm{Y}_{\mathrm{ijkl}}=\mu+\alpha_{\mathrm{i}}+\beta_{\mathrm{j}}+\gamma_{\mathrm{k}}+\alpha \beta_{\mathrm{ij}}+\alpha \gamma_{\mathrm{ik}}+\beta \gamma_{\mathrm{jk}}+\alpha \beta \gamma_{\mathrm{ijk}}+\varepsilon_{\mathrm{ijk}}$, où $\mathrm{Y}=$ variable, teneur en azote, $\mu=$ moyenne, $\alpha=$ effet du type de fourrage, $\beta=$ effet de l'état du fourrage, $\gamma=$ effet de la durée de conservation et $\varepsilon=$ effet de l'erreur.

Le second essai a porté sur les chaumes de maïs (Zea mays) et les chaumes de mil (Pennisetum thyphoides) qui provenaient des exploitations agricoles locales. Le matériel et les méthodes utilisés ont été les mêmes que pour le premier essai.

\section{RESULTATS ET DISCUSSION}

\section{Premier essai}

Les effets du type de fourrage et du séchage sur la teneur en azote de la paille de brousse et des chaumes de sorgho traités à 4,5 p. 100 d'urée sont présentés au tableau I. Le type de fourrage a eu un effet significatif $(\mathrm{P}<0,05)$ sur la fixation de l'azote. Avec le traitement à 4,5 p. 100 d'urée, les teneurs en azote de la paille de brousse et des chaumes de sorgho sont passées respectivement de 0,55 à 1,49 et de 0,33 à 1,40 p. 100 .
Le séchage a diminué la teneur en azote de 1,84 à 1,05 g pour $100 \mathrm{~g}$ d'aliment (soit une réduction de 43 p. 100). Ceci s'explique par la perte d'ammoniac au cours du séchage. Le séchage de la paille de riz traitée à 4,5 p. 100 d'urée diminue la teneur en azote de 40 p. 100 (5). L'interaction aliment * état a été significative $(\mathrm{P}<0,01)$. Le séchage a eu un effet négatif plus marqué $(\mathrm{P}<0,01)$ sur les chaumes de sorgho $(1,88$ à 0,92 p. 100) que sur la paille de brousse $(1,80$ à 1,18 p. 100).

L'effet de la durée de conservation sur la teneur en azote des fourrages pauvres traités à 4,5 p. 100 d'urée est présenté au tableau II. La durée de conservation a eu un effet négatif $(\mathrm{P}<0,05)$ sur la teneur en azote de la paille de brousse et des chaumes de sorgho traités à 4,5 p. 100 d'urée. La comparaison des moyennes effectuée par la méthode de Ppds a donné les résultats suivants :

- la teneur en azote à un jour de conservation a été supérieure $(\mathrm{P}<0,05)$ à celle des autres durées de conservation ;

- la teneur en azote est restée constante $(\mathrm{P}>0,05) \mathrm{du} 15^{\mathrm{e}}$ au $90^{\mathrm{e}}$ jour de conservation.

La teneur élevée en azote à un jour de conservation s'est expliquée par l'absence d'activité uréolitique, d'où la présence de l'azote sous sa forme initiale, celle de l'urée. Ces résultats sont conformes à ceux rapportés par Hadjipanayiotou (1). A partir du 15 jour, la teneur en azote total a diminué et s'est stabilisée. Ce phénomène s'explique par l'hydrolyse progressive de l'urée en ammoniac sous l'effet de l'activité des enzymes végétales et bactériennes puis des pertes d'azote ammoniacal qui se produisent à l'ouverture du silo. Hadjipanayiotou rapporte un taux de fixation de 44 p. 100 dont 31 p. 100 sous forme d'azote ammoniacal (1).

L'effet de l'interaction aliment * durée de conservation a été significatif $(\mathrm{P}<0,05)$. La teneur en azote de la paille de brousse est restée la plupart du temps plus élevée que celle des chaumes de sorgho. Les effets du type d'aliment, du séchage et de la durée de conservation sont présentés à la figure 1 : tout au long de l'essai,

\section{Tableau I}

Effets du type de fourrage et du séchage sur la teneur en azote de la paille de brousse et des chaumes de sorgho traités à 4,5 p. 100 d'urée

\begin{tabular}{lcc} 
& Paille de brousse & Chaumes de sorgho \\
\hline Humides & $1,80 \pm 0,36$ & $1,88 \pm 0,27$ \\
Séchés & $1,18 \pm 0,37$ & $0,92 \pm 0,34$ \\
Moyenne & $1,49 \pm 0,48^{a}$ & $1,40 \pm 0,58$ b
\end{tabular}

a, $\mathrm{b}$ : les chiffres de la même colonne ou de la même ligne suivis de lettres différentes sont différents au seuil de 5 p. 100

\section{Tableau II}

Effets de la durée de conservation sur la teneur en azote de la paille de brousse et des chaumes de sorgho traités à 4,5 p. 100 d'urée

\begin{tabular}{lccccccc} 
& $\mathbf{1}$ jour & $\mathbf{1 5}$ jours & $\mathbf{3 0}$ jours & $\mathbf{4 5}$ jours & $\mathbf{6 0}$ jours & $\mathbf{7 5}$ jours & $\mathbf{9 0}$ jours \\
\hline Paille de brousse & 2,17 & 1,54 & 1,41 & 1,29 & 1,46 & 1,28 & 1,28 \\
Chaumes de sorgho & 1,91 & 1,42 & 1,44 & 1,53 & 1,25 & 1,13 & 1,12 \\
Moyenne & 2,04 a & $1,48 \mathrm{~b}$ & $1,43 \mathrm{~b}$ & $1,41 \mathrm{~b}$ & $1,35 \mathrm{~b}$ & $1,21 \mathrm{~b}$ & $1,20 \mathrm{~b}$
\end{tabular}


la teneur en azote de la paille de brousse traitée humide et des chaumes de sorgho traités humides est restée plus élevée que celle de la paille de brousse traitée séchée et des chaumes de sorgho traités séchés. La diminution de la teneur en azote a été plus prononcée pour les fourrages secs que pour les fourrages humides.

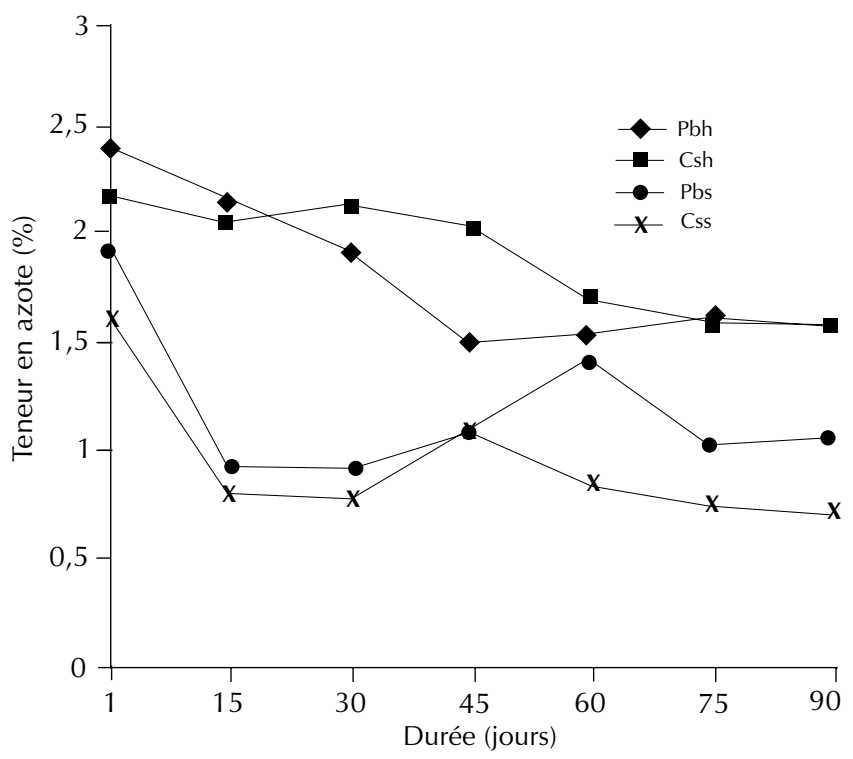

Figure 1 : effets du type de fourrage et de la durée de conservation sur la teneur en azote de la paille de brousse et des chaumes de sorgho traités à 4,5 p. 100 d'urée. Pbh = paille de brousse humide, Csh = chaumes de sorgho humides, $\mathrm{Pbs}=$ paille de brousse séchée, $C s s=$ chaumes de sorgho séchés.

\section{Deuxième essai}

L'effet du type de fourrage et du séchage sur la teneur en azote des chaumes de maïs et des chaumes de mil traités à 4,5 p. 100 d'urée est présenté au tableau III. Les teneurs initiales en azote ont été respectivement de 0,47 et 0,46 p. 100 pour les chaumes de mil et les chaumes de maïs. Le type de fourrage n'a pas eu d'effet significatif $(\mathrm{P}>0,05)$ sur la fixation d'azote. La teneur en azote a été respectivement de 1,28 et 1,30 g pour $100 \mathrm{~g}$ de fourrage pour les chaumes de maïs et de mil traités à 4,5 p. 100 d'urée. Ces teneurs ont été légèrement inférieures à celles obtenues au cours du premier essai.

Le séchage a réduit la concentration d'azote de 27 p. 100 (1,48 à $1,08 \mathrm{~g} \mathrm{~N} / 100 \mathrm{~g}$ de fourrage). La diminution a été respectivement de 23 et 30 p. 100 pour les chaumes de mil et de maïs.

L'effet de la durée de conservation sur la teneur en azote des fourrages traités est présenté au tableau IV. La durée de conservation a eu un effet significatif $(\mathrm{P}<0,05)$ sur la fixation d'azote des chaumes de maïs et de mil. Comme lors du premier essai, la teneur en azote à un jour de conservation a été supérieure à celle des autres durées de conservation et, à partir du $15^{\mathrm{e}}$ jour, la teneur en azote total est restée constante $(\mathrm{P}>0,05)$ jusqu'au $90^{\mathrm{e}}$ jour de conservation.

Les effets du séchage, du type de fourrage et de la durée de conservation des chaumes de maïs et de mil sont présentés à la figure 2 . La diminution de la teneur en azote a été plus prononcée pendant les quinze premiers jours pour les deux types de fourrages. La perte d'azote par séchage a eu lieu pendant toute la durée de l'essai comme le montrent les courbes des chaumes de maïs et de mil séchés comparées aux courbes des chaumes de maïs et de mil humides.

\section{Tableau III}

Effets du type de fourrage et du séchage sur la teneur en azote des chaumes de maïs et des chaumes de mil traités à 4,5 p. 100 d'urée

$\begin{array}{lccc} & \text { Chaumes de maïs } & \text { Chaumes de mil } & \text { Moyenne } \\ \text { Humides } & 1,49 \pm 0,43 & 1,47 \pm 0,33 & 1,48 \pm 0,38^{\mathrm{a}} \\ \text { Séchés } & 1,04 \pm 0,30 & 1,13 \pm 0,44 & 1,08 \pm 0,37^{\mathrm{b}} \\ \text { Moyenne } & 1,28 \pm 0,41^{\mathrm{a}} & 1,30 \pm 0,38^{\mathrm{a}} & \end{array}$

a, b : les chiffres de la même colonne ou de la même ligne suivis de lettres différentes sont différents au seuil de 5 p. 100

\section{Tableau IV}

Effets de la durée de conservation sur la teneur en azote des chaumes de maïs et des chaumes de mil traités à 4,5 p. 100 d'urée

\begin{tabular}{lcccccccc} 
& $\mathbf{1}$ jour & $\mathbf{1 5}$ jours & $\mathbf{3 0}$ jours & $\mathbf{4 5}$ jours & $\mathbf{6 0}$ jours & $\mathbf{7 5}$ jours & $\mathbf{9 0}$ jours \\
\hline Chaumes de maïs & 2,02 & 0,99 & 1,11 & 1,03 & 1,17 & 1,18 & 1,32 \\
Chaumes de mil & 2,16 & 1,16 & 1,17 & 1,13 & 1,18 & 1,10 & 1,19 \\
Moyenne & 2,09 a & $1,07^{b}$ & $1,14 b$ & 1,08 b & $1,18^{b}$ & $1,14^{b}$ & 1,25 b
\end{tabular}

$a, b$ : les chiffres de la dernière ligne suivis de lettres différentes sont différents au seuil de 5 p. 100 


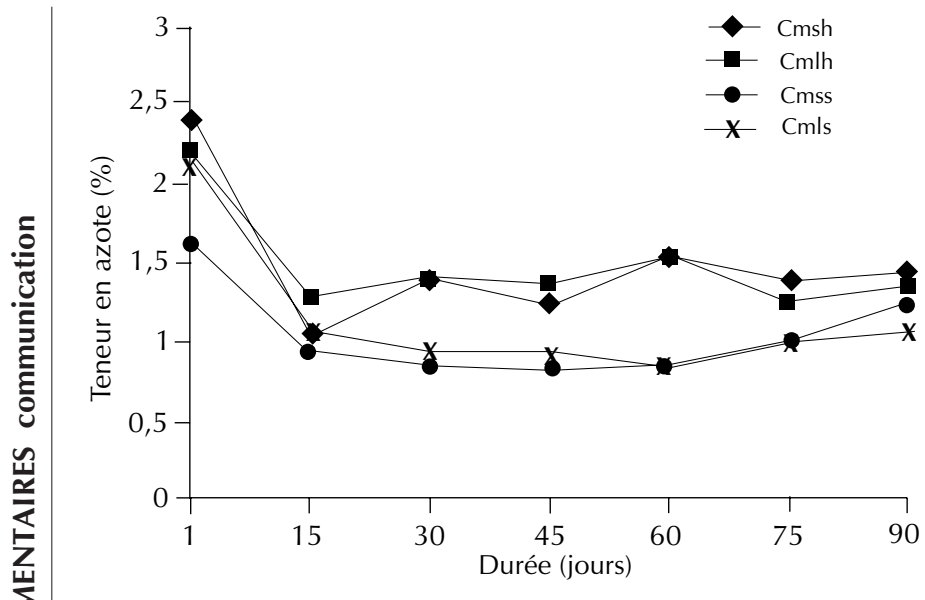

Figure 2 : effets du type de fourrage et de la durée de conservation sur la teneur en azote des chaumes de maïs et de mil traités à 4,5 p. 100 d'urée. Cmsh = chaumes de maïs humides, $\mathrm{Cm} / \mathrm{h}=$ chaumes de mil humides, Cmss = chaumes de maïs séchés, $\mathrm{Cm} / \mathrm{s}=$ chaumes de mil séchés.

\section{CONCLUSION}

Le traitement des fourrages pauvres avec 4,5 p. 100 d'urée a amélioré la teneur en azote de 114 à 178 p. 100. Le séchage a réduit la teneur en azote $(\mathrm{P}<0,05)$ des fourrages traités de 27 à 43 p. 100 . La teneur en azote a été plus élevée $(\mathrm{P}<0,05)$ à un jour de conservation. A partir du $15^{\mathrm{e}}$ jour et jusqu'au $90^{\mathrm{e}}$ jour, les variations de la teneur en azote des fourrages traités ont été insignifiantes $(\mathrm{P}>$ $0,05)$.

Dans les conditions climatiques de l'étude, quinze jours ont suffi pour le traitement des fourrages pauvres et la teneur en azote des fourrages traités est restée élevée pour une durée de conservation de 15 à 90 jours. Le séchage a diminué la teneur en azote des fourrages pauvres traités, d'où une préférence d'utilisation des fourrages à l'état humide pour minimiser les pertes d'azote.

5. SIVAKUMAR M.V.K., KONATE M., VIRMANI S.M., 1984. Agroclimatology of West Africa: Mali. Patancheru, India, ICRISAT, 294 p. (Information Bulletin No. 19)

6. TRAORE S.M., KASSAMBARA I., 1989. L'amélioration de la valeur alimentaire des fourrages pauvres : la paille traitée à l'urée et séchée ou la paille traitée complémentée par le bloc melur. Bamako, Mali, Inrzfh, 13 p. (Com. tech. Prod. Anim.)

7. WAGEPETERSEN J., VESTERGAARD T.K., 1977. Effect on digestibility and nitrogen content of barley straw of different ammonia treatments. Anim. Feed Sci. Tech., 2: 131-142.

Accepté le 01.08.2001

\section{Resumen}

Nantoumé H., Kouriba A., Ouologuem B. Efectos de la duración de la conservación y del secado sobre el contenido de nitrógeno en pastos pobre tratados con urea

En el marco de un experimento de 90 días, concebido para el estudio de los efectos de la duración de la conservación y del secado sobre el contenido de nitrógeno en forrajes pobres (paja de tamojal, rastrojo de sorgo, rastrojo de maíz y rastrojo de trigo) tratados a 4,5\% con urea, se utilizaron bocales como silos de laboratorio. El contenido de nitrógeno en los forrajes tratados permaneció significativamente más elevado $(\mathrm{p}<$ $0,05)$ que el de los no tratados, cualquiera que fuese la duración de la conservación. El secado disminuyó el contenido de nitrógeno $(p<0,05)$ de los forrajes tratados. Una vez que el tratamiento fue efectivo (15 días), el contenido de nitrógeno de los forrajes tratados no varió $(p>0,05)$ durante el estudio. El estudio demuestra que es más ventajoso ofrecer forrajes tratados no secos, con el fin de minimizar las pérdidas de nitrógeno durante el secado.

Palabras clave: Forraje - Contenido de nitrógeno - Urea Aptitud para la conservación - Secado - Calidad - Malí. 